\title{
R\&D ERL: Beam dynamics, parameters and physics to be learned
}

D. Kayran

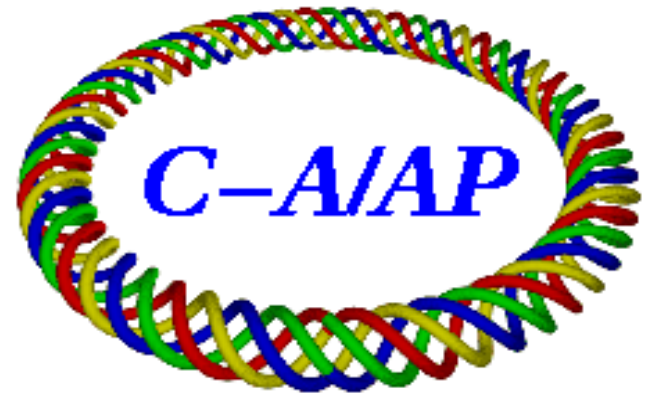

\section{Collider-Accelerator Department Brookhaven National Laboratory Upton, NY 11973}

Notice: This document has been authorized by employees of Brookhaven Science Associates, LLC under Contract No. DE-AC02-98CH10886 with the U.S. Department of Energy. The United States Government retains a nonexclusive, paid-up, irrevocable, world-wide license to publish or reproduce the published form of this document, or allow others to do so, for United States Government purposes. 


\title{
R\&D ERL: Beam dynamics, parameters and physics to be learned.
}

\author{
D. Kayran, for BNL R\&D ERL group
}

\section{Introduction}

The R\&D ERL facility at BNL aims to demonstrate CW operation of ERL with average beam current in the range of 0.1-1 ampere, combined with very high efficiency of energy recovery. The ERL is being installed in one of the spacious bays in Bldg. 912 of the RHIC/AGS complex (Fig. 1). The bay is equipped with an overhead crane. The facility has a control room, two service rooms and a shielded ERL cave. The control room is located outside of the bay in a separate building. The single story house is used for a high voltage power supply for $1 \mathrm{MW}$ klystron. The two-story unit houses a laser room, the CW $1 \mathrm{MW}$ klystron with its accessories, most of the power supplies and electronics

The ERL R\&D program has been started by the Collider Accelerator Department (C-AD) at BNL as an important stepping-stone for 10-fold increase of the luminosity of the Relativistic Heavy Ion Collider (RHIC) using relativistic electron cooling [1] of gold ion beams with energy of $100 \mathrm{GeV}$ per nucleon. Furthermore, the ERL R\&D program extends toward a possibility of using $10-20 \mathrm{GeV}$ ERL for future electron-hadron/heavy ion collider, MeRHIC/eRHIC [2]. These projects are the driving force behind the development of ampere-class ERL technology, which will find many applications including light sources and FELs.

The intensive R\&D program geared towards the construction of the prototype ERL is under way: from development of high efficiency photo-cathodes [3] to the development of new merging system compatible with emittance compensation [4].

Table 1: ERLs parameters under development at BNL

\begin{tabular}{|c|c|c|c|c|c|c|c|}
\hline & \multicolumn{2}{|c|}{$\begin{array}{l}\text { R\&D ERL } \\
\text { design }\end{array}$} & \multicolumn{5}{|c|}{ BNL ERL projects requirements } \\
\hline & \begin{tabular}{|l|} 
High \\
Current \\
\end{tabular} & \begin{tabular}{|l} 
High \\
charge
\end{tabular} & PoP CeC & Test *) & $\begin{array}{l}\text { Pre-cooling } \\
\text { a } 40 \mathrm{GeV}\end{array}$ & MeRHIC & $\begin{array}{l}\text { eRHIC } \\
10 / 20 \\
\end{array}$ \\
\hline Charge per bunch, $\mathrm{nC}$ & 0.7 & 5 & 5 & 5 & $14(9 \times 1.56)$ & 5 & $18 / 3.5$ \\
\hline Energy maximum/injection, $\mathrm{MeV}$ & $20 / 2.5$ & $20 / 3.0$ & $21 / 3$ & $21 / 3$ & $21 / 3$ & $4000 / 10$ & $\begin{array}{l}10000 / 10 \\
20000 / 10\end{array}$ \\
\hline $\begin{array}{l}\text { R.m.s. Normalized emittances } \\
\text { ex/ey, } \mathrm{mm}^{*} \mathrm{mrad}\end{array}$ & $1.4 / 1.4$ & $4.8 / 5.3$ & 5 & 5 & 3 & $7-73$ & 77 \\
\hline R.m.s. Energy spread, dE/E & $3.5 \times 10-3$ & $1 \times 10^{-2}$ & $1.5 \times 10^{-3}$ & $1.5 \times 10^{-3}$ & $810^{-4}$ & $2 \times 10^{-3}$ & $1 \times 10^{-3}$ \\
\hline R.m.s. Bunch length, ps & 18 & 31 & 30 & 30 & 30 & 6.7 & 30 \\
\hline Bunch rep-rate, $\mathrm{MHz}$ & 700 & 9.383 & 0.078 & 9.383 & 9.383 & 9.383 & 14.1 \\
\hline Gun/dumped avrg. current, mA & 500 & 50 & 0.4 & 50 & 130 & 50 & 50 \\
\hline Linac average current, $\mathrm{mA}$ & 1000 & 100 & 0.4 & $0.4 / 50$ & 130 & 300 & 500 \\
\hline Injected/ejected beam power, MW & 1.0 & 0.150 & 0.0012 & 0.15 & 0.390 & 0.5 & 0.5 \\
\hline Numbers of passes & 1 & 1 & 1 & 1 & 1 & 3 & 5 \\
\hline
\end{tabular}

\section{Goals for ERL R\&D at BNL}

R\&D ERL will serve as a test-bed for future RHIC projects (Table 1):

- ERL-based electron cooling (conventional or coherent). 
- $\quad$ 10-to-20 GeV ERL for lepton-ion collider eRHIC.

Test the key components of the High Current ERL based solely on SRF technology

SRF Photoinjector (703.5 MHz SRF Gun, photocathode, laser, merger etc.) test with $500 \mathrm{~mA}$.

- Preservation of high-charge, low emittance.

High current 5-cell SRF linac test with HOM absorbers

- Single turn - $500 \mathrm{~mA}$

Stability criteria for CW beam current.

Attainable ranges of electron beam parameters in SRF ERL.

\section{Layout of the R\&D ERL}

The schematic ERL design (shown in Fig. 2) has one turn: electrons are generated in the superconducting half-cell gun and injected into the main superconductive linac. Linac accelerates electrons $15-20 \mathrm{MeV}$, which pass through a one turn re-circulating loop with achromatic flexible optics [5].

The photocathode is located in a high electric field for immediate acceleration of the electrons to as high energy as possible, reducing emittance degradation due to strong space charge force. Furthermore, liner part of space charge effects is compensated by applying a suitable external solenoid magnetic field.

In nominal recovery operation regime the path-length of the loop provides for 180 degrees change of the RF phase, causing electron deceleration (hence energy recovery) down to injection energy. The decelerated beam separates from the higher energy beam and goes to the beam-dump.

\section{R\&D ERL modes of operation}

Two operating modes are envisaged, namely the high current mode and the high charge mode. The high current $(0.5 \mathrm{~A})$ mode will operate electron bunches with lower normalized emittance, $0.7 \mathrm{nC}$ charge per bunch at $703 \mathrm{MHz}$ rep-rate. In this case, the full energy of electrons at gun exit is limited by the available RF power $2.5 \mathrm{MeV}$. In high charge mode electron beam will consist of bunches with charge up to $5 \mathrm{nC}$ per bunch at $10 \mathrm{MHz}$ repetition rate, $50 \mathrm{~mA}$ average current. The electrons energy at the exit of the gun can be pushed up to 3.0-3.5 MeV by the maximum field attainable in the super-conducting gun itself. 


\section{ERL in BNL bldg 912}

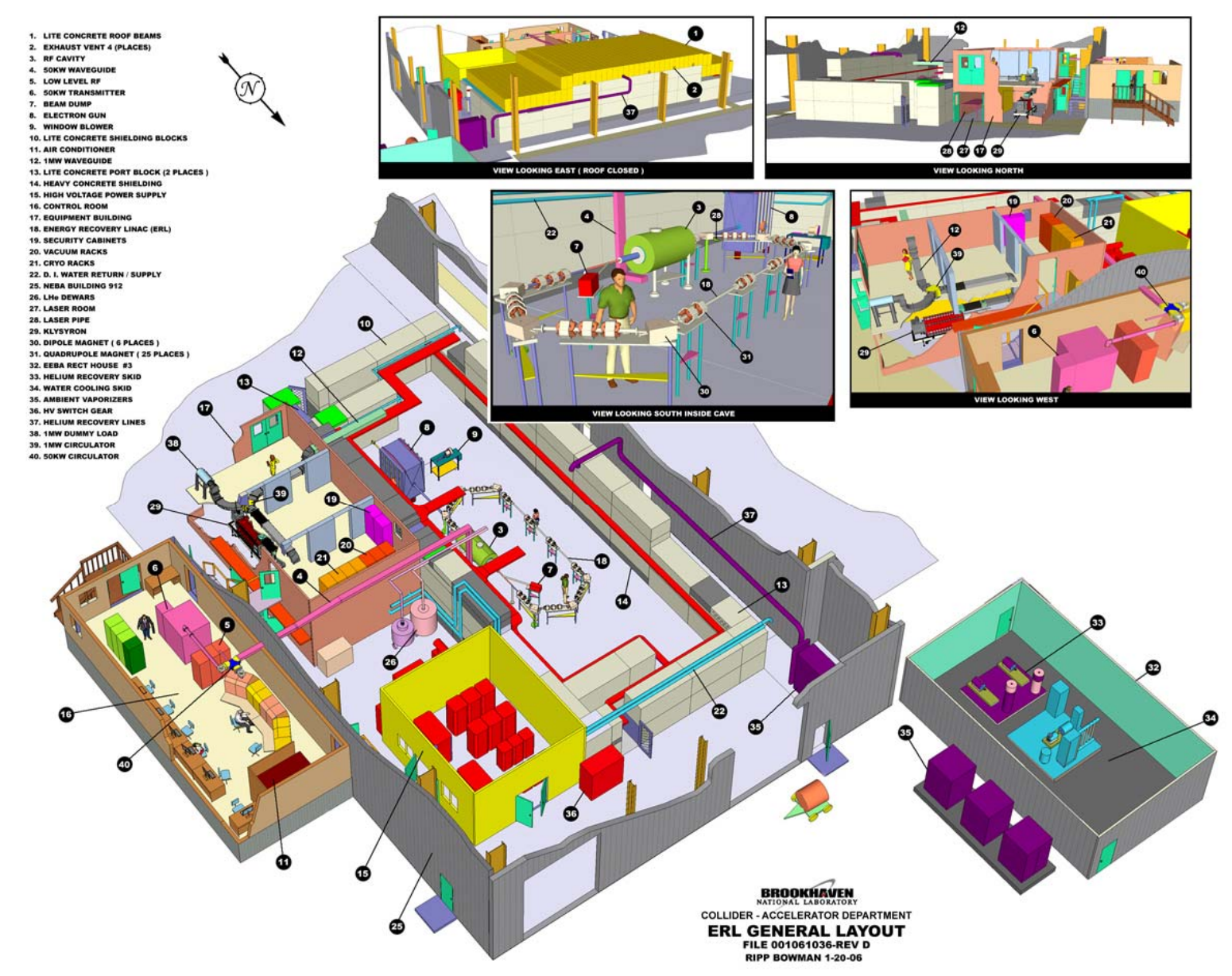

Figure 1: The layout of the R\&D ERL facility in the bay of Bldg. 912 at BNL. 


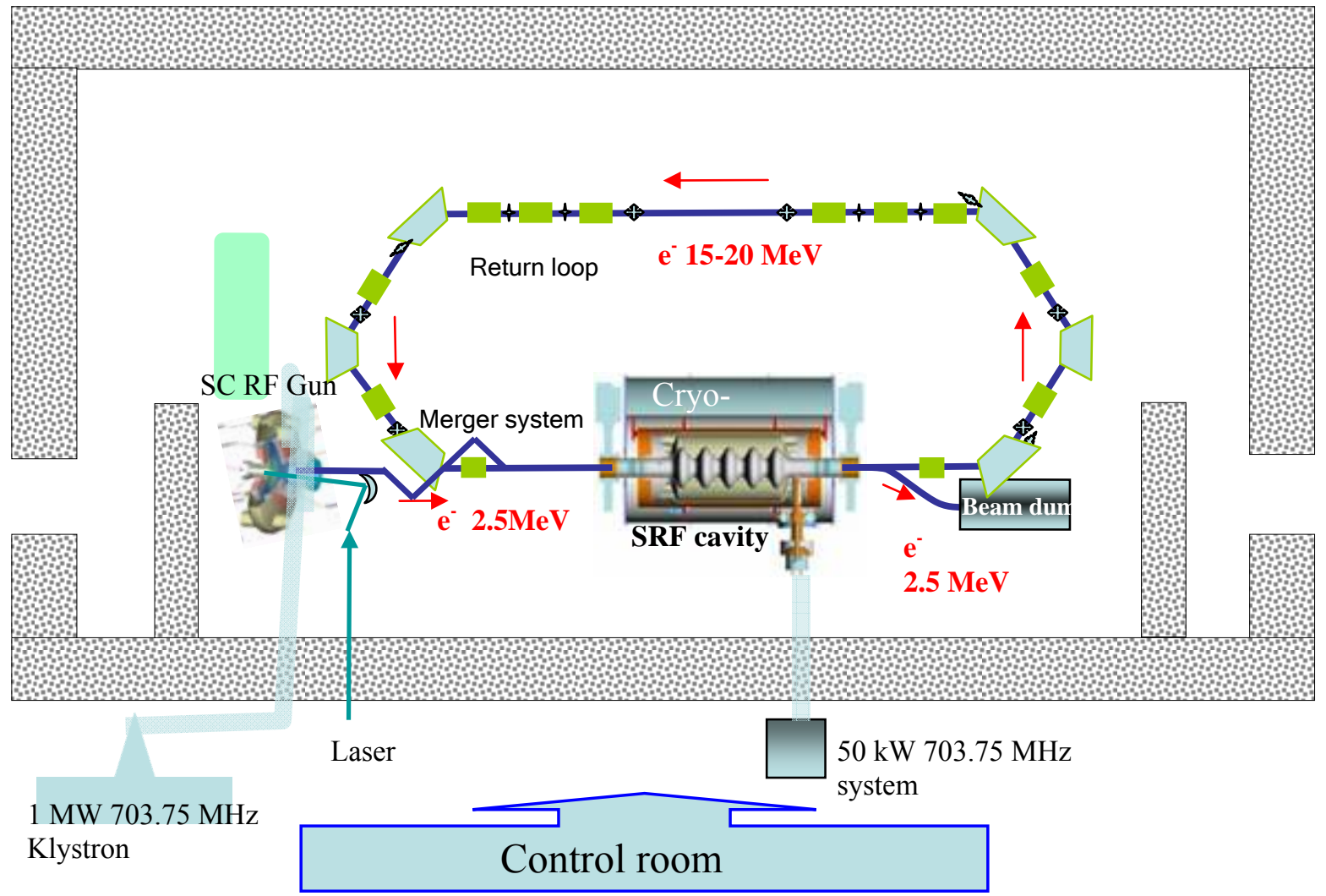

Figure 2: Layout of the R\&D energy recovery linac in the shielded vault in bldg 912. :

Table 2: Beam modes.

\begin{tabular}{|l|l|l|l|}
\hline Beam mode & Bunch charge $[\mathrm{nC}]$ & Rep rate $[\mathrm{MHz}]$ & Beam current $[\mathrm{mA}]$ \\
\hline Tune up & $0.1-1.4$ & $10-6-9.383$ & $10-7-14$ \\
\hline Low charge & 1.4 & 9.383 & 14 \\
\hline High current & $1.4 / 0.7$ & $351.88-703.76$ & 500 \\
\hline High charge & 5 & 9.383 & 50 \\
\hline
\end{tabular}

\section{BNL R\&D ERL SRF Injector}

The electron injector is a central part of any ERL that has to deliver high brightness electron beam. The BNL R\&D ERL injector [6] (see Fig. 3) consist of $1 / 2$ cell superconducting RF gun with photocathode inside, solenoid, four dipoles and two solenoids turned on in opposite direction (in order to match the electron beam with linac entrance more accurately). The $4^{\text {th }}$ dipole mergers the high and low energy beams.

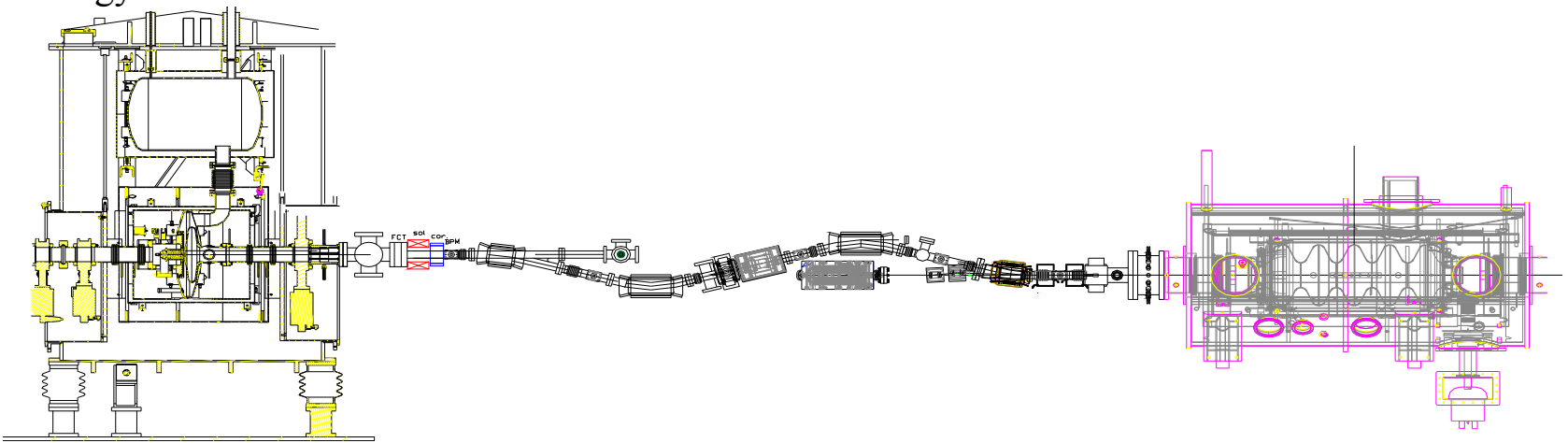

Figure 3: Detailed drawing of SRF Injector for the BNL R\&D ERL. 


\section{Half cell SRF Gun}

The frequency of the gun is chosen $703.75 \mathrm{MHz}$, or the $75^{\text {th }}$ harmonic of the $9.383 \mathrm{MHz}$ bunch spacing frequency of RHIC. To operate in CW mode with $500 \mathrm{~mA}$ current and $2 \mathrm{MeV}$ kinetic energy beam the gun should supply about $1 \mathrm{MW}$ power in to the beam. Low RF power losses in superconducting RF (SRF) gun and high peak electric field near the cathode followed by the emittance compensation scheme make SRF guns ideal injectors for high current low emittance applications.

There are various projects which right now are considering the use of SRF photo-injector. It seems that in three to four years time the SRF Gun will become a routinely running injector for high current low emittances applications.

For R\&D ERL the superconducting 703.75 MHz RF (SRF) gun was selected (Fig.4). The gun design with a short $8.5 \mathrm{~cm}$ cell was chosen in order to provide reasonably high electric field at the cathode at this low accelerating voltage. To provide effective damping of high order mode (HOM) this gun has rather large iris radius of $5 \mathrm{~cm}$. More details on the $\mathrm{SRF}$ gun and its photocathode system can be found elsewhere [7].

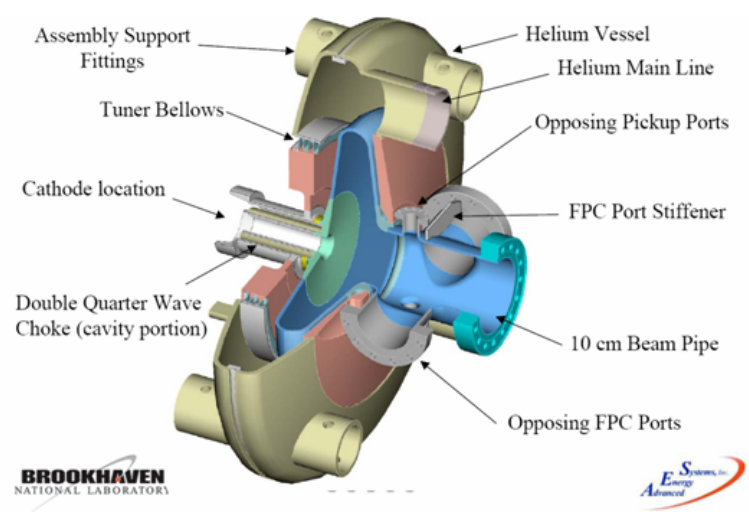

Figure 4: The SRF gun, in helium vessel and choke joint cathode insertion port. The high power fundamental power coupler (FPC) ports are also shown (left). The 703 SRF Gun fabricated by AES under HOM studies (right)

To keep the beam from the growing in size shortly after being emitted from the cathode, a focusing element in close proximity to the cathode is very desirable. A cathode recess provides an electric RF focusing near a cathode region where the space charge force is most significance. The HTS solenoid is located in $35 \mathrm{~cm}$ from the cathode surface to keep beam size from increasing and to launch the emittance compensation mechanism [8].

The performance of a SRF photo-injector has been studied using SUPERFISH (to calculate the electric and magnetic fields) by PARMELA [9] (to calculate the beam dynamics).

\section{Cathode position}

The photocathode is located in a high electric field for immediate acceleration of the electrons to as high energy as possible, reducing emittance degradation due to strong space charge force. Furthermore, liner part of space charge effects is compensated by applying a suitable external solenoid magnetic field.

The $1 / 2$ cell gun shape with recessed cathode is shown on Fig. 2. A consequence of the cathode recess is that the accelerating field at the cathode is reduced by about $20 \%$ a factor with $3 \mathrm{~mm}$ recessed and can be almost flat with $+1 \mathrm{~mm}$ move into the cavity (Fig. 5.). 

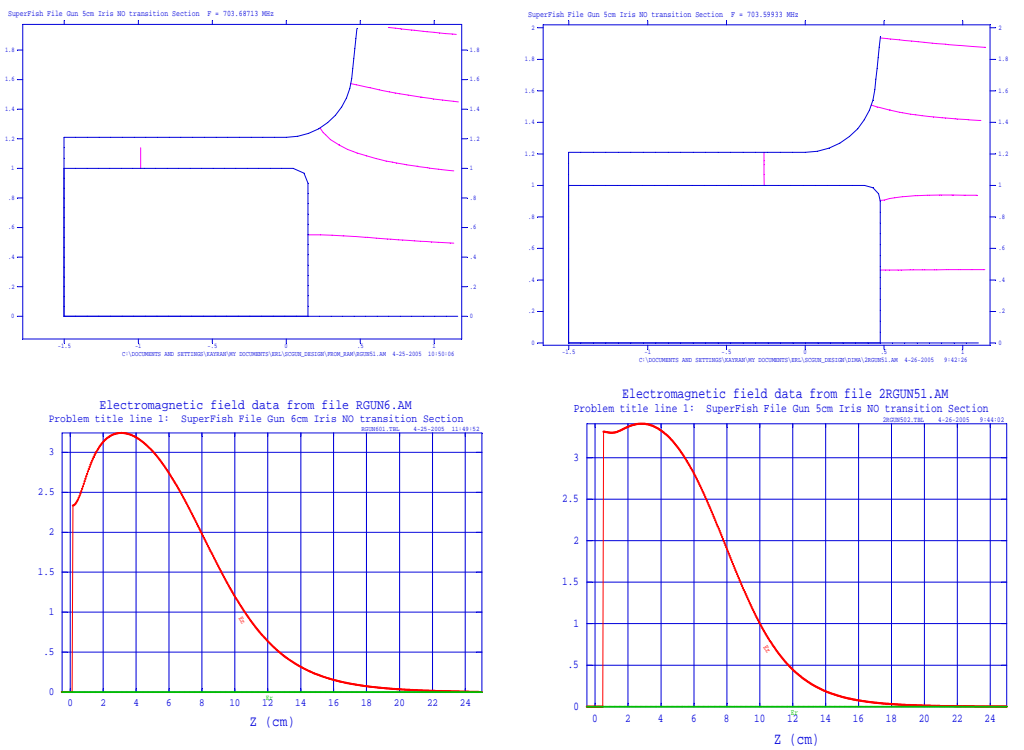
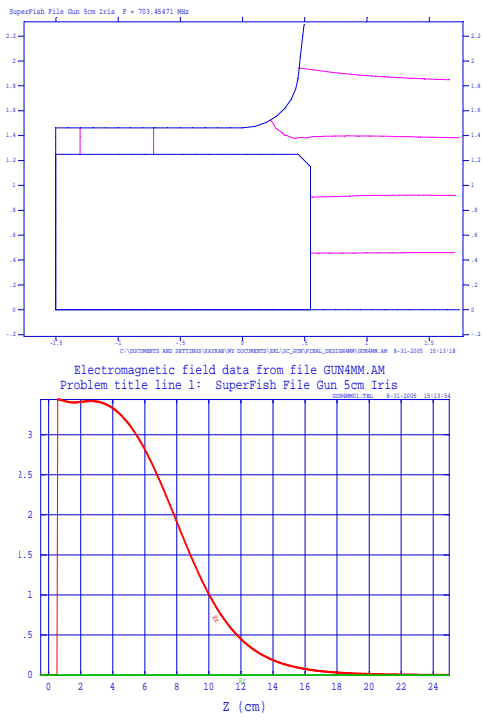

Figure 5: The cathode location (top) and electric field on the axis (bottom) (results of the SUPERFISH simulation) for different recess of the cathode: $-3 \mathrm{~mm}$ (left), $0 \mathrm{~mm}$ (center) $+1 \mathrm{~mm}$ (right).

The actual amount of recess must be determined by optimizing the effects of the larger electric field on the cathode which favor no recess, and transverse focusing which favors a recess [10]. We used a simplified injection system (without bends) to study the cathode position effect and launch phase on both longitudinal and transversal dynamics (Fig. 6). For each recess position of the cathode, the initial spot size, bunch length, and the solenoids were adjusted to minimize emittance at the exit of the Linac.

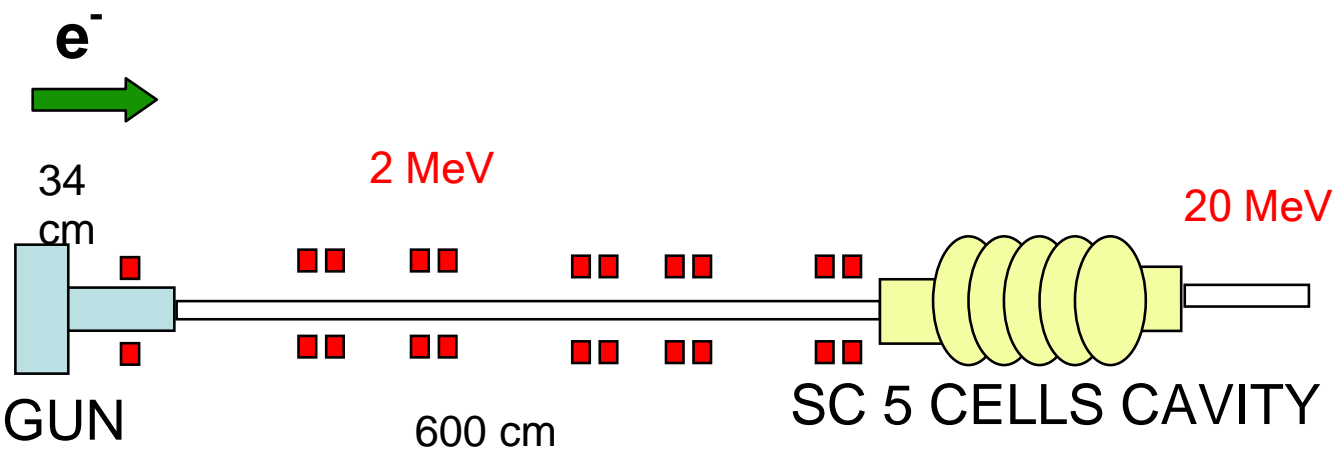

Figure 6: The injection test-bed without bends to study cathode recess/ launch phase dependences. It can be seen from Fig. 7 that position of the cathode with respect the cavity wall has a strong influence on both longitudinal and transverse emittances. The launch phase for a fixed recess was varied \pm 5 degrees which had a weak effect on the final emittances. Therefore, the cathode positions of $-1 \pm 0.5 \mathrm{~mm}$ with respect to a cavity wall seems to be an optimal region, and an adjustable cathode stalk is proposed for the prototype design to determine the best insertion length with beam 


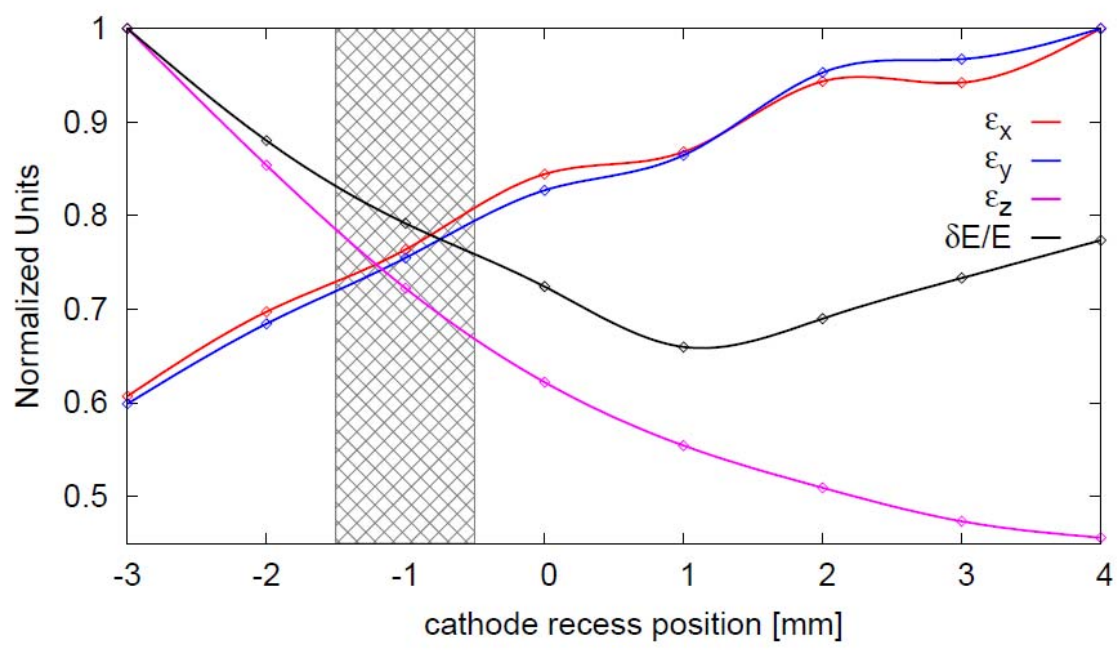

Figure 7: Longitudinal and transverse emittances at the end of a $20 \mathrm{MeV}$ Linac for different positions of the cathode in SRF gun (results of PARMELA simulations). The energy of the e- at the exit of the gun was fixed. Note that all emittances are normalized and the solid lines are spline fits to the simulation points.

\section{Longitudinal beam dynamics}

Longitudinal motion is determined by the initial phase at the cathode, accelerating voltage and the space charge. Space charge increases a negative energy chirp from head to tail of the electron bunch and makes it longer. For the first year of Prototype ERL operation there is no plan to use any boosterchopper RF cavity in injection line. Only a natural slope of the energy gain versus the launch phase and ballistic compression can be used to prevent electron beam from lengthening. Fig. 8 shows the energy gain in the gun as a function of the initial phase of the electrons. Results of PARMELA simulation for energy spread and bunch length evolutions $(0.7 \mathrm{nC}$ per bunch) for two different initial phases are shown in Fig. 9.

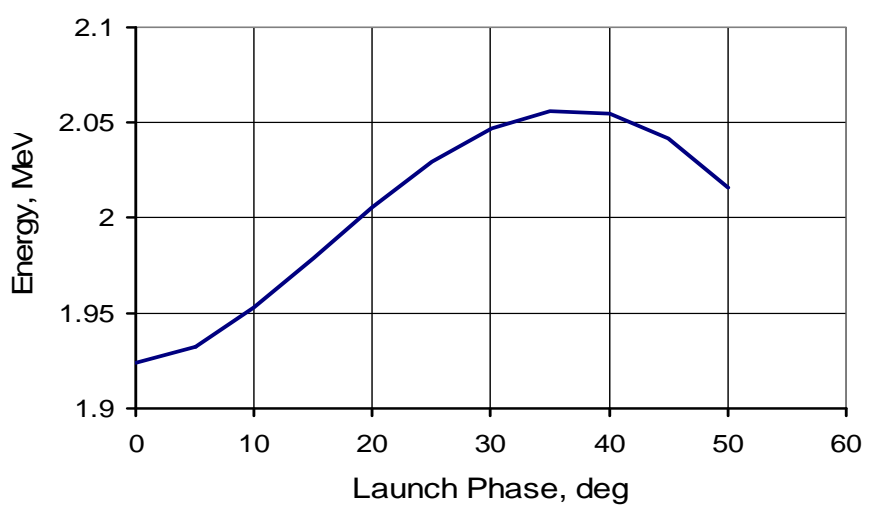

Figure 8: Electron beam energy gain at the exit of the gun versus initial phase 

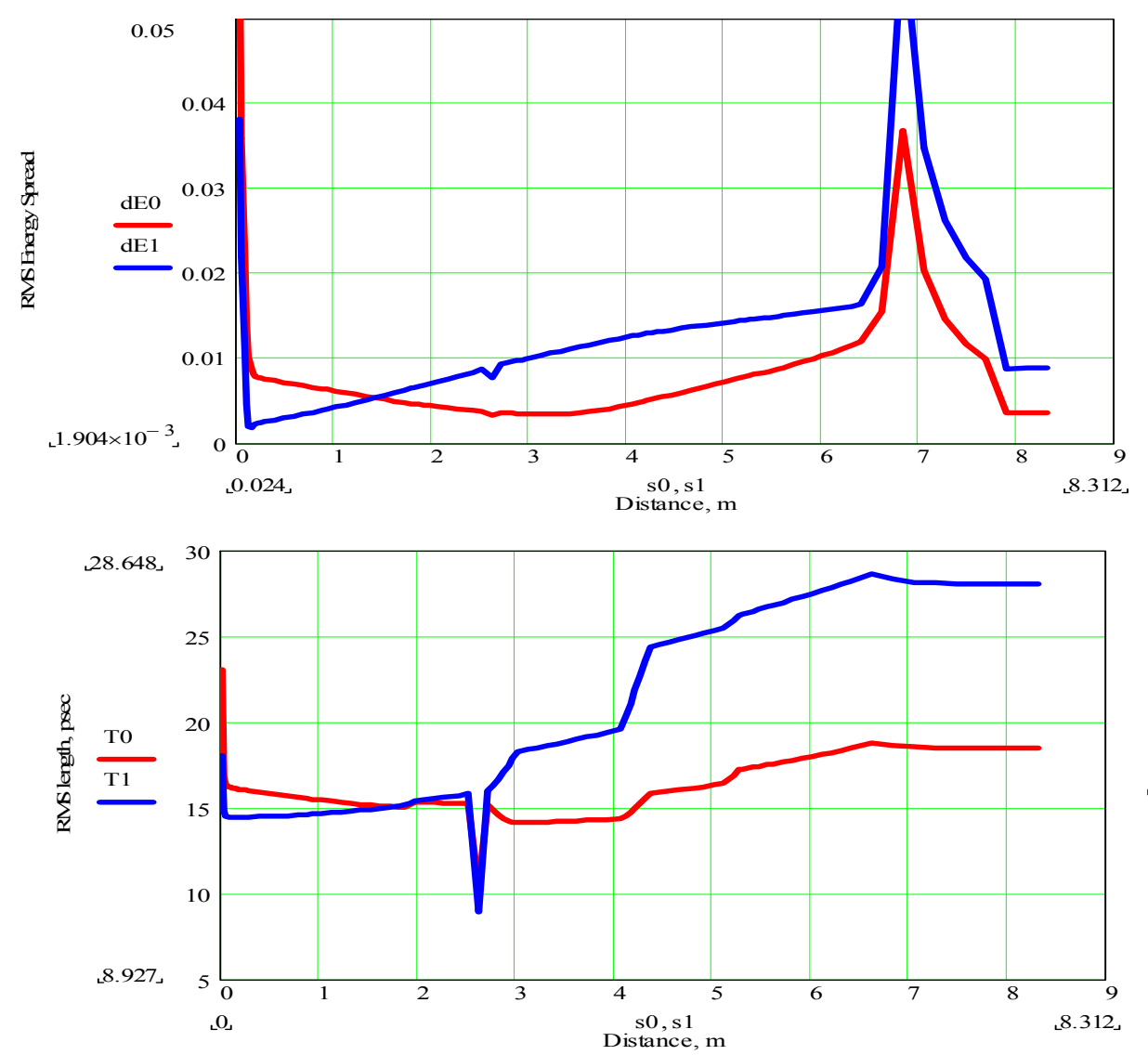

Figure 9: RMS energy spread (top figure) and rms bunch length (bottom figure) as a function of distance from the cathode for two different launch phases $25 \mathrm{deg}-\mathrm{RED}$ and $35 \mathrm{deg}-\mathrm{BLUE}$

\section{Merger system}

One of the critical parts of the ERL injector is the merger of the low energy- and high energy beams (Fig. 10). The injection energy is not recovered. Low injection energy requires less RF power and lowers dumped beam energy. The original emittance compensation scheme [8] does not include any dipoles between RF gun and linac (or booster cavity).

One of the novel systems we plan to use for the R\&D ERL is a merging system providing achromatic condition for space charge dominated beam and compatible with the emittance compensation scheme [4].

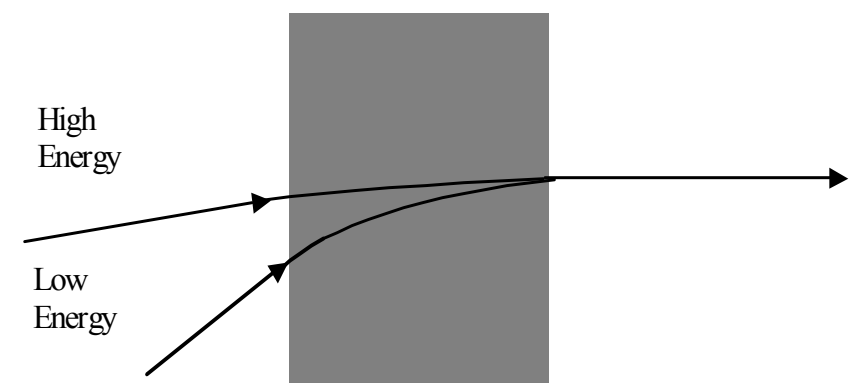

Figure 10: Main function of a merger - combining two (or more) beams with different energies.

Focusing of the bending magnets in the merging section has significant effect on the low energy electrons. Different focusing in vertical and horizontal planes (astigmatism) makes impossible simultaneous emittance compensation. Hence, the use of combined function magnets [11] with 
equal focusing strength in $\mathrm{x}$ - and $\mathrm{y}$ - direction is necessary. The merger must be designed to minimize degradation of the low energy beam emittance.

Any dipole magnet excites the coupling between energy and transverse motion which leads to effective correlated emittance growth:

$$
\varepsilon_{x}^{e f f}=\sqrt{\varepsilon_{x}^{2}+\sigma_{x}^{2} D^{\prime 2} \sigma_{\delta}^{2}+\sigma_{x^{\prime}}^{2} D^{2} \sigma_{\delta}^{2}},
$$

where: $D$-is dispersion, $D^{\prime}$-is dispersion derivative, $\varepsilon_{x}$ is emittance without dispersion and $\sigma_{x}, \sigma_{x^{\prime}}, \sigma_{\delta}$ are rms size, angular spread and energy spread respectively.

The full merging system has to decouple such correlations at the exit (i.e. full decupling of longitudinal and transverse motions). There are many systems which work very well in the absence of space charge effects: chicane, dogleg, achromatic bend etc.

Basically it means that the achromatic system has to satisfy two traditional conditions:

$$
\int_{s_{o}}^{s_{f}} K_{o}(s) \cdot m_{12}\left(s \mid s_{f}\right) \cdot d s=0 ; \int_{s_{o}}^{s_{f}} K_{o}(s) \cdot m_{22}\left(s \mid s_{f}\right) \cdot d s=0,
$$

where $K_{0}(s)$-is curvature of trajectory, $s_{0}, s, s_{f}$ - are initial, current and final positions respectively along the transport system $m_{12}\left(s \mid s_{f}\right), m_{22}\left(s \mid s_{f}\right)$ - are (1-2) and (2-2) elements of 6x6 transport matrix from $s$ to $s_{f}$ position.

In presence of a space charge effect the particles energy is changing during the passing through the merger system. In this case to decouple transverse and longitudinal motions the additional two conditions have to be satisfied:

$$
\int_{s_{o}}^{s_{f}} K_{o}(s) \cdot s \cdot m_{12}\left(s \mid s_{f}\right) \cdot d s=0 ; \int_{s_{o}}^{s_{f}} K_{o}(s) \cdot s \cdot m_{22}\left(s \mid s_{f}\right) \cdot d s=0 .
$$

One of the possible merging schemes which satisfied both pairs of conditions and preserves the emittance of the low energy is shown in Fig. 2. This Z-system provides a minimum set of elements (4 magnets) for the decupling. The detailed description of the Z-system, its principles of operation and comparison different merger systems can be found in [4]. Fig. 11 shows result of PARMELA simulations of the ERL injector for different charge per bunch.
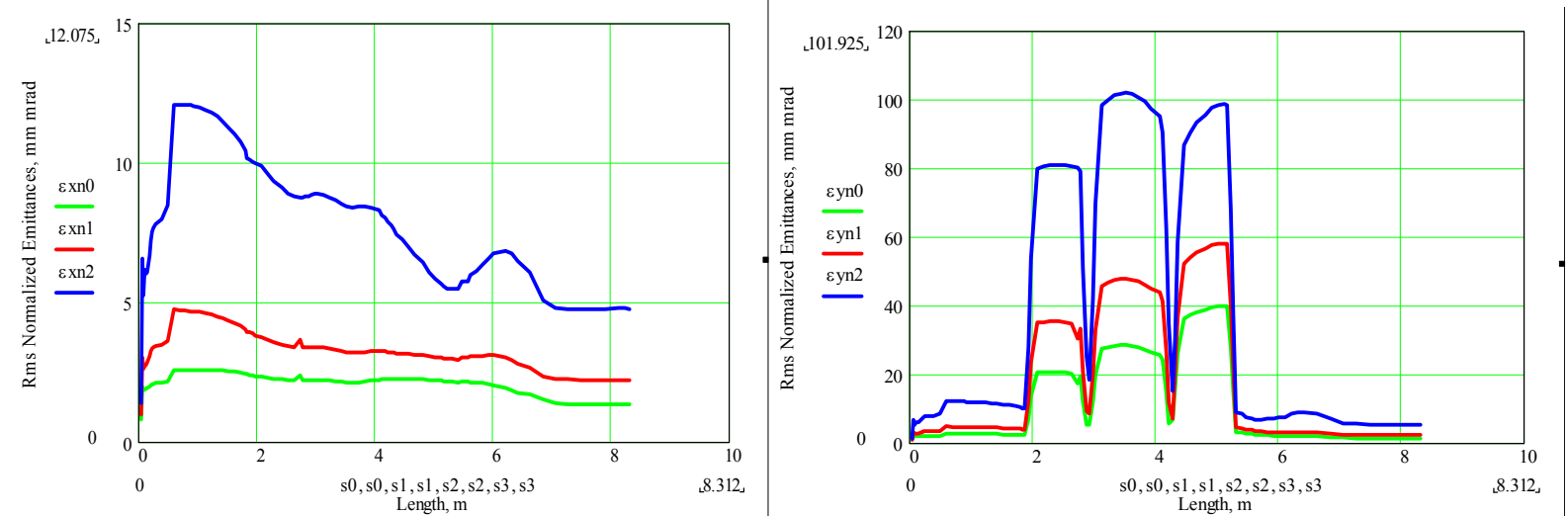

Figure 11: Evolution of normalized beam emittances (top figure - horizontal, bottom figurevertical) in the ERL injector. Bunch charge: $0.7 \mathrm{nC}$-GREEN, $1.4 \mathrm{nC}$ - RED, 5nC -BLUE. Due to the bends in vertical direction the effect of vertical emittance growth is clear. But at the exit of Zmerger both: vertical and horizontal emittances become almost equal. In case of $5 \mathrm{nC}$ per bunch this equality is broken, the next order nonlinearity start playing a role.

The main expected electron beam parameters of this system obtained by PARMELA simulations are listed in Table 3. 


\section{Injection combine function magnets}

The beam size in injection line can reach as large as $7 \mathrm{~mm}$ RMS size the high order multiples from focusing elements can increase emittance.

Due to very small real estate and large beam size: each magnet includes 4 sets of coils: 1) vertical bend, 2) quadrupole focusing, 3) sextupole correction and 4) horizontal steering.

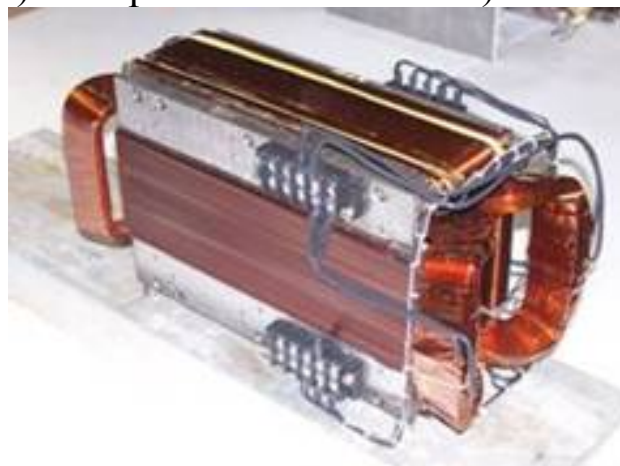

Figure 12. Window frame multifunctional injection dipole.

The quadrupole coil is used to split focusing equally between the planes

The amount of the sextupole component is controlled by the gap between the yoke and the main dipole coil. A small additional coil in the corners is a sextupole trim coil, intended for use if sextupole component needs to be adjusted to reduce emittance growth.

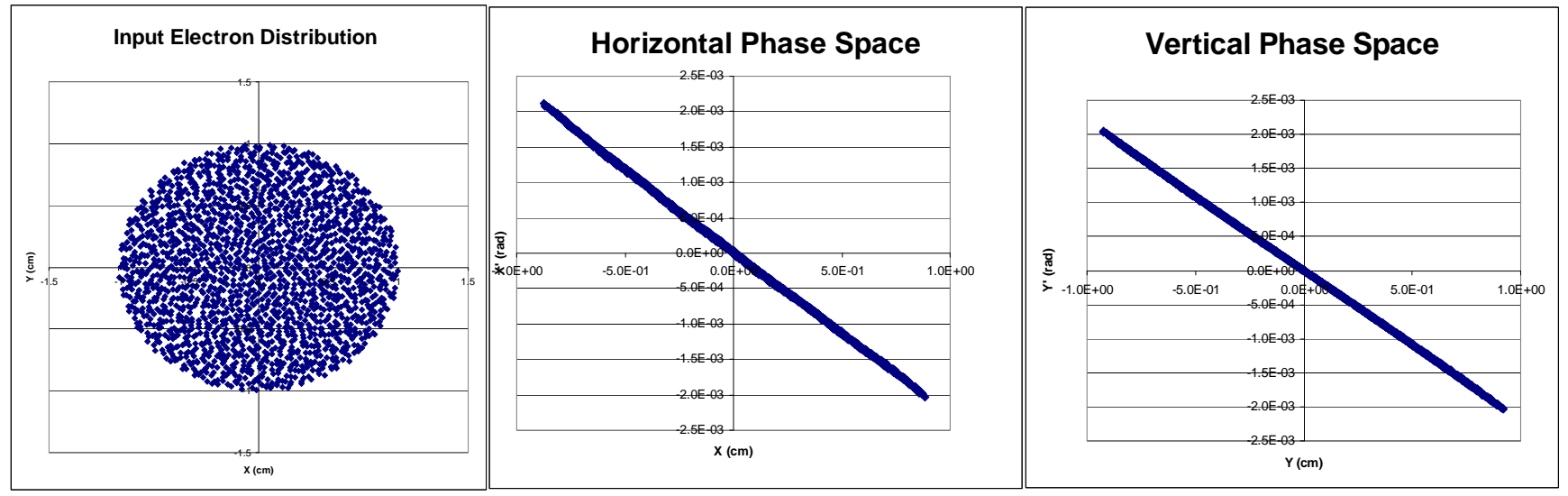

Figure 13: Result of tracking particles by W. Meng using Opera3d in the magnetic field map initial emittances 0 . After tracking normalized emittance is $0.6 \mu$.

Analysis predicts that the influence of various field components on the emittance growth is complicated by the fact that the beam trajectory bends significantly in the fringe fields. Hence, direct tracking in the calculated fields extracted from Opera3d was used of test beam to evaluate and to minimize influence of magnetic field on the beam emittance (Fig 13) [11].

\section{Injector summary}

The injector for BNL R\&D ERL can produce ampere electron beam with low emittance. High brightness injector will serve as an electron source for the prototype ERL. The results of the design studies of the R\&D ERL and PARMELA simulation are very promising. The main expected electron beam parameters of this system are listed in Table 3.

Optimization of the transverse beam emittance and the longitudinal beam emittance for BNL R\&D ERL injector results in different launch phases. We plan to use both modes of operation. 
Table 3: Electron beam parameters of the R\&D ERL .

\begin{tabular}{|l|l|l|l|}
\hline Charge per bunch, $\mathrm{nC}$ & 0.7 & 1.4 & 5 \\
\hline Injection energy, $\mathrm{MeV}$ & 2.5 & 2.5 & 3 \\
\hline Max. beam energy, $\mathrm{MeV}$ & 20 & 20 & 20 \\
\hline Average beam current, $\mathrm{A}$ & 0.5 & 0.5 & 0.05 \\
\hline Bunch rep-rate, $\mathrm{MHz}$ & 700 & 350 & 9.38 \\
\hline Normalized emittance ex/ey, $\mu \mathrm{m}$ & $1.4 / 1.4$ & $2.2 / 2.3$ & $4.8 / 5.3$ \\
\hline Rms energy spread, \% & 0.35 & 0.5 & 0.97 \\
\hline Rms bunch length $\mathrm{ps}$ & 18.5 & 21 & 31 \\
\hline
\end{tabular}

\section{ERL: returning loop}

The list of requirements for ERL returning path are summarized in Table 4.

Table 4: Requirements for ERL prototype loop lattice

Modes of operation

High average current + high brightness

High charge per bunch

High energy acceptance

Beam break up instability study

Longitudinal motion study

Two pass acceleration
Critical parameters

$\mathrm{I}=0.5 \mathrm{~A}$, en $<5 \mathrm{~mm} * \mathrm{mrad}$

$\mathrm{Q}=0.7-5 \mathrm{nC}$

Low dispersion, large aperture

Adjustable transverse phase advance

Adjustable longitudinal dispersion

Changeable path length

In order to change the phase of the second pass thought the linac from 0 to 90 degrees then 180 degrees the path length has to be changed. The wave length of main harmonic RF is $43 \mathrm{~cm}$ it seems quite difficult to design a compact chicane to adjust the phase in such a large range. In R\&D ERL design the first arc is installed on movable stand and there are two long bellows in the straight sections of vacuum chamber. The movement of the arc from $+/-6 \mathrm{~cm}$ (see Fig. 14)

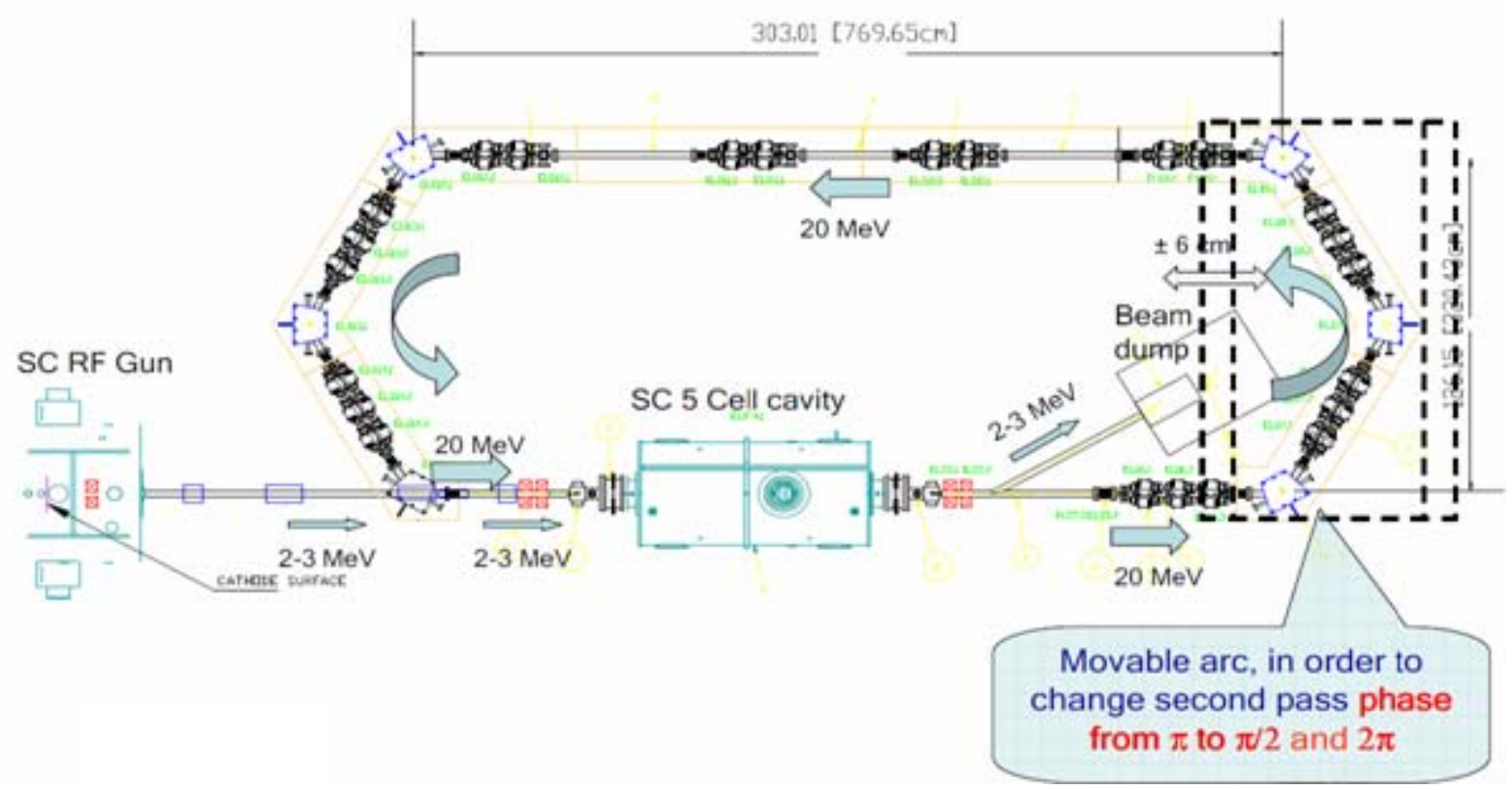


Figure 14: R\&D ERL layout with indication of movable arc.

\section{Loop lattice}

The lattice of the ERL loop controls the parameters of a symplectic transport matrix, which affect the stability and operation conditions of the ERL. The lattice of the loop is intentionally chosen to be very flexible for the R\&D ERL to be a test-bed of new ampere-range of beam currents in ERL technology. The adjustable part of the lattice has two arcs and a straight section. Each arc is an achromatic with adjustable longitudinal dispersion. Quadrupoles in the dispersion-free straight section provides for matching of the $\beta$-function and for choosing the desirable phase advances independently in horizontal and vertical planes. The lattice functions for different longitudinal momentum compaction are shown in Fig.15. The PARMELA tracking result for isochronous regime and $0.7 \mathrm{nC}$ per bunch from the cathode to the dump for are shown in Fig.16 and Fig. 17.

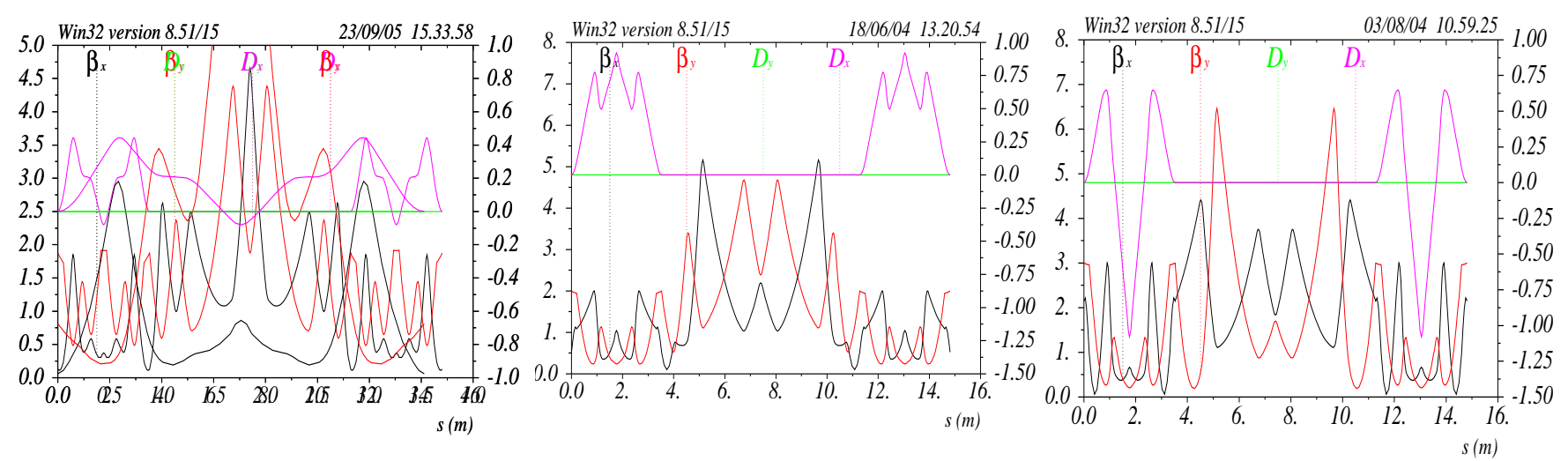

Figure 15: Lattice $\beta$ and D functions of the ERL for the different cases longitudinal dispersions (Ds=M56): zero (left), positive (center), negative (right).

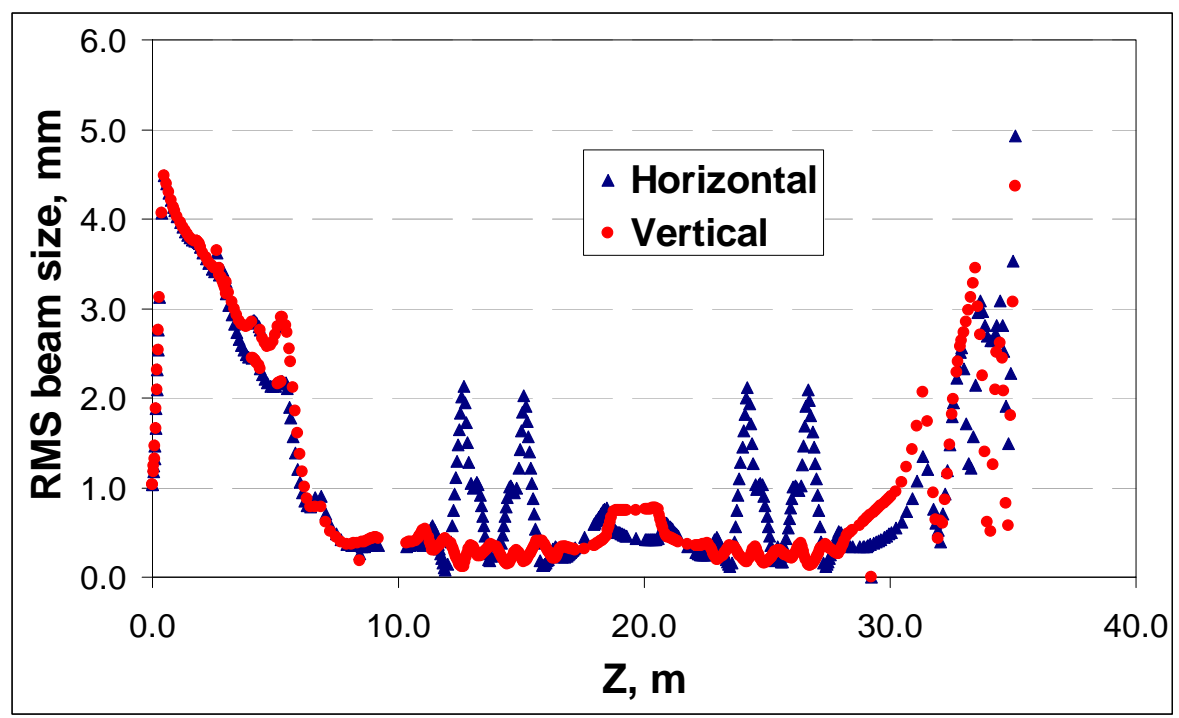

Figure 16: RMS beam size evolution from the cathode to the beam dump as a PARMELA tracking $(\mathrm{Q}=0.7 \mathrm{nC}$ per bunch). 


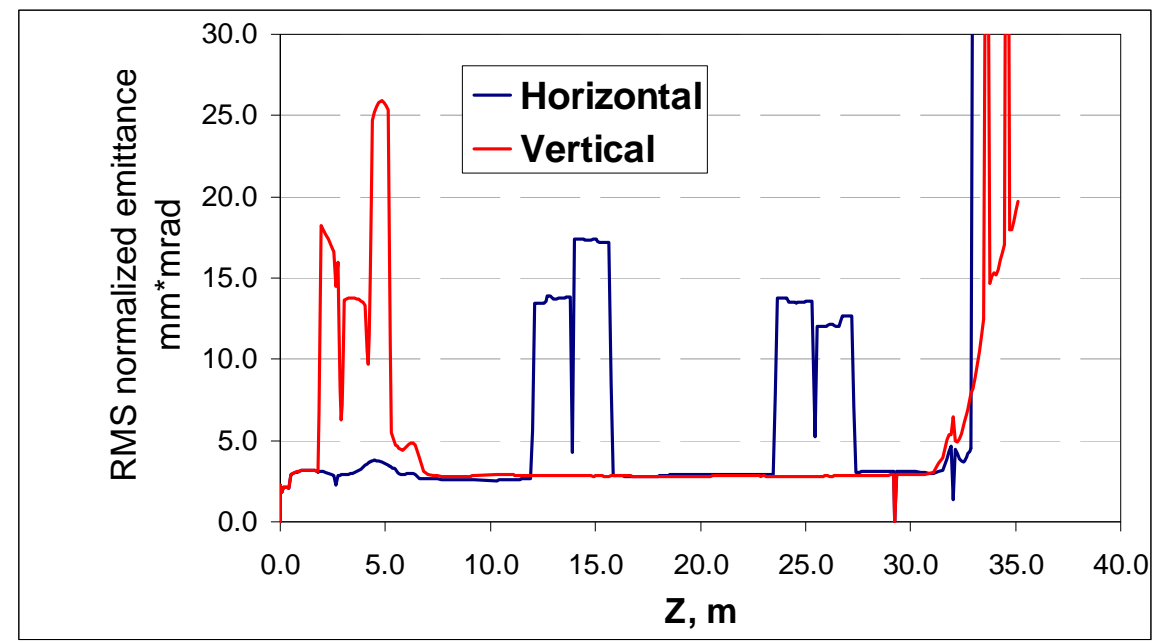

Figure 17: RMS normalized emittances evolution from the cathode to the beam dump as a PARMELA tracking $(\mathrm{Q}=0.7 \mathrm{nC}$ per bunch).

The PARMELA simulation demonstrates what after the acceleration to $20 \mathrm{MeV}$ the emittances are preserved (Fig. 17). There are effective horizontal emittance jumps due to nonzero dispersion inside the banding arcs. However in dispersion free straight section vertical and horizontal emittances are equal again.

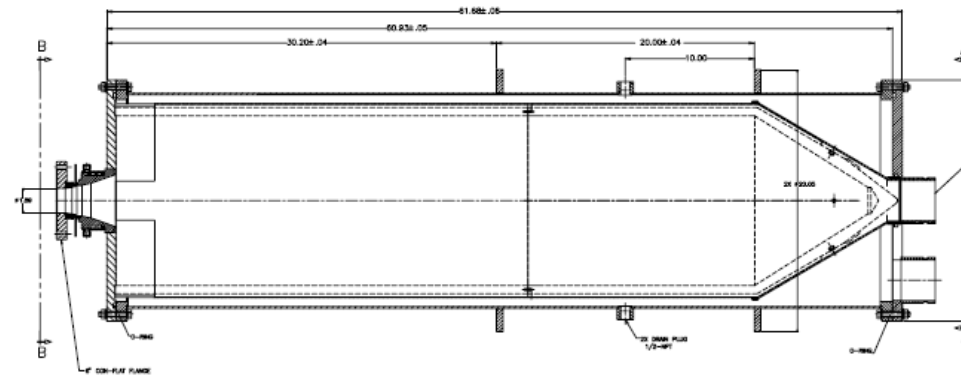

Figure 18: 1MW CPI Klystron beam collector accepts 1MW $2 \mathrm{MeV}$ beam
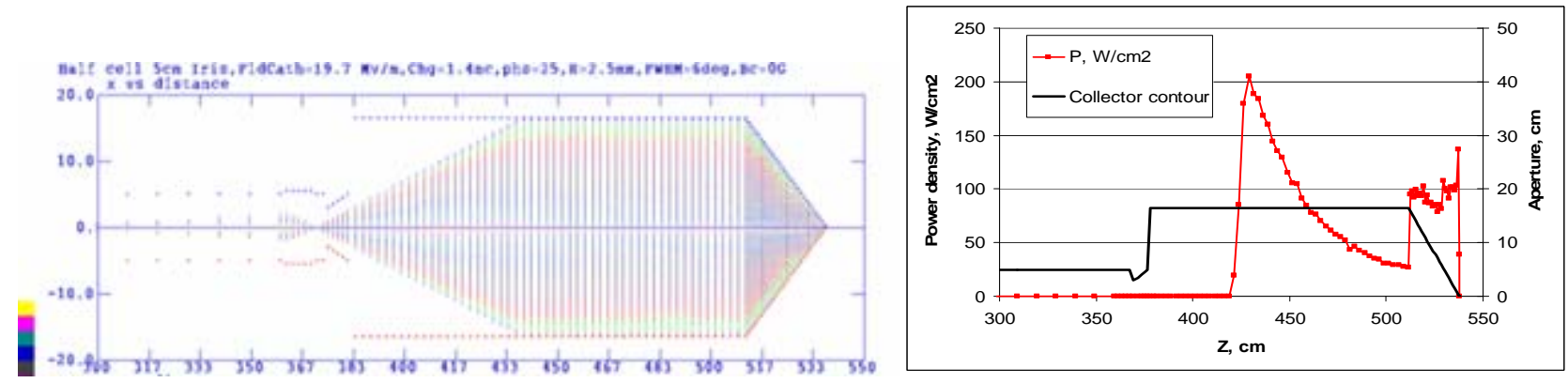

Figure 19: Particle trajectory (left) and power density distribution (right).of $1 \mathrm{MW}$ electron beam in the 1MW CPI Klystron beam collector

\section{Beam dump}

After a cycle of acceleration and deceleration back to the injection energy $2.5 \mathrm{MeV}$ electron beam goes to beam dump. The beam dump has bullet like shape inside with full water cooled jacket around. This beam dump is an exact copy of that is used for $1 \mathrm{MW}$ klystron water cooled collector (Fig 18) and this beam dump can accept electron up to $2 \mathrm{MW}$ average power CW. In order to avoid very hot spots on a surface of the beam dump the electron beam is over focused by short focal length solenoid. For $2.5 \mathrm{MeV}$ energy beam the $10 \mathrm{~cm} 2.7 \mathrm{kGauss}$ solenoid was used in simulations. 
The result of simulation shows on Fig 19 what maximum power density is less $200 \mathrm{~W} / \mathrm{cm}^{2}$.

\section{Sextupole correction in arcs}

The second order effects on beam parameters in arcs due to large energy spread were simulated by E. Pozdeyev [12] using program DIMAD. While the vertical emittance is almost constant, the horizontal one significantly changes due to the energy spread (Fig.20). The horizontal emittance increases twice after passing two arcs.

It was shown that after using sextupoles corrections in arcs (Fig. 21.) the emittance shows almost no growth in both directions (Fig. 9)
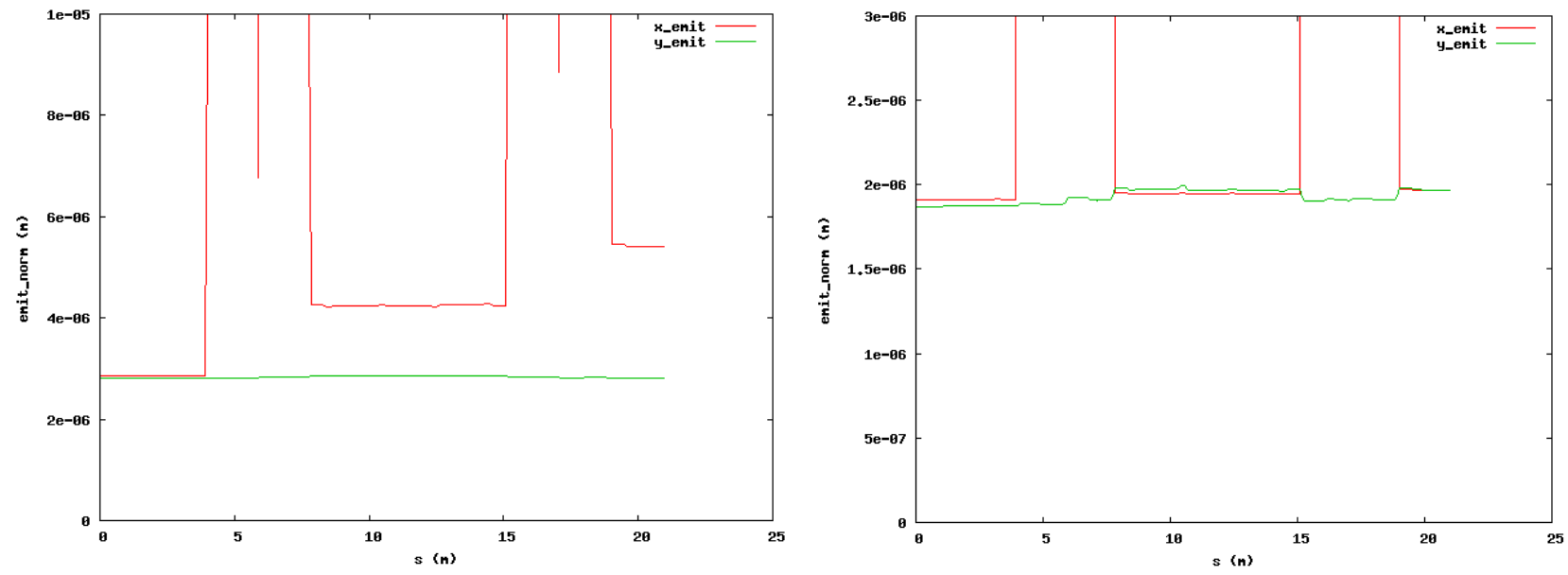

Figure 20: Vertical and horizontal r.m.s. beam emittances as a function of the path-length: without second order correction (left) and with second order correction (right).

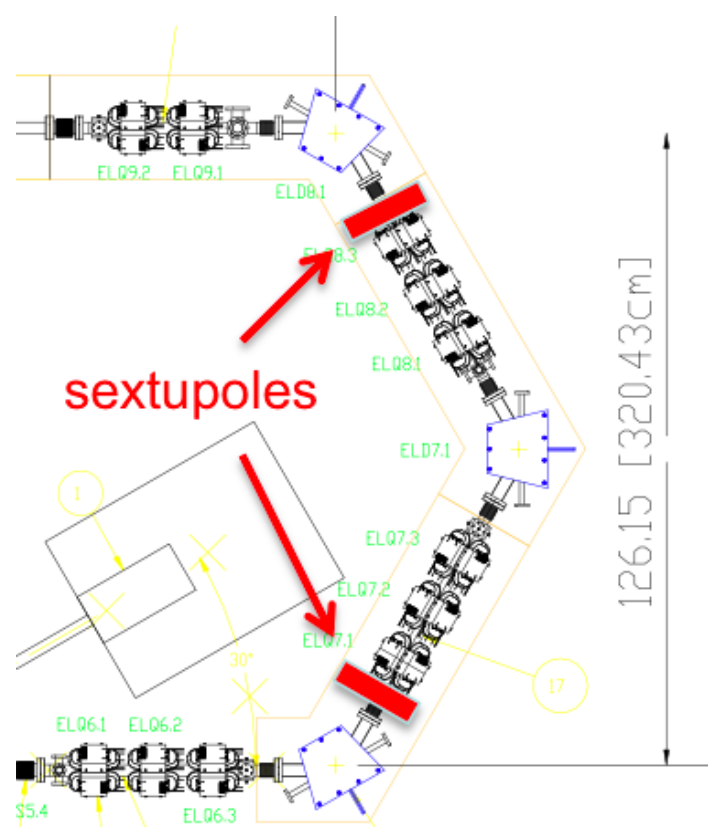

Figure 21: ERL arc with two identical sextupoles compensating second order in the straight section 


\section{COLLECTIVE EFFECTS}

In the ERL, collective effects can be separated into two groups: instabilities that can limit the maximum beam current and effects that tend to dilute the beam quality. The former group includes transverse cumulative and multi-pass beam breakup (BBU) instabilities. The latter group includes the space charge effect, wake fields, CSR in bending magnets, trapped ions. Some of these effects will be covered in next chapter some of them still need to be addressed.

\section{Beam Break Up Instability}

The current two-loop design of the electron cooler ERL utilizes two SRF cavities. Because of the cavities number is small, the cumulative (single-pass) BBU presents no danger in the R\&D ERL. Transverse multi-pass BBU can present an insurmountable problem for operations of an ERL. The design of the 5-cell SC BNL cavity [7] was optimized to reduce the quality factor of HOMs and push BBU threshold beyond the ampere level.

If there is only one high order mode and one recirculation pass the value of BBU threshold current can be calculated using the formula [13]:

$$
I_{t h}=-\frac{2 c^{2}}{e(R / Q)_{\omega} Q_{\omega}} \frac{1}{m^{*} \sin \left(\omega t_{r}\right)},
$$

where $\omega$ is the frequency of dipole $\mathrm{HOM}, R / Q_{\omega}$ is the shunt impedance, $Q_{\omega}$ is the quality factor, $c$ is the speed of light, $e$ is the elementary charge, $m^{*}$ is a combination of the transport matrix elements:

$m^{*}=m_{12} \cos ^{2} \theta_{\omega}+\left(m_{14}+m_{32}\right) \cos \theta_{\omega} \sin \theta_{\omega}+m_{34} \sin ^{2} \theta_{\omega}$, where $m_{i j}$ is the recirculation matrix elements, $\theta_{\omega}$ is the polarization angle, $\mathrm{t}_{\mathrm{r}}$ - is bunch return time.

In case of many modes and/or many passes ERL the computer simulations to find the threshold have to be performed.

The BBU threshold current for the R\&D ERL was simulated using codes GBBU [14]. The Qs of dipole HOMs are taken from the measurements of the 5cell cavity at bldg 912 and R/Q values were simulated by CST Microwave Studio [15].

For the optics with zero longitudinal dispersion in the arcs (base line optics) the value of the threshold current more then $20 \mathrm{~A}$ is well above of operational 50mA or even $500 \mathrm{~mA}$.

Next the effect of quadrupole HOMs to the BBU needs to be calculated.

\section{Coherent Synchrotron Radiation (CSR)}

The r.m.s. energy spread and the average particle energy loss, ignoring vacuum chamber shielding, can be estimated from the following expressions:

$$
\begin{aligned}
& \sigma_{E}=0.25 \frac{r_{e} N_{e} L_{e f f}}{\gamma\left(R^{2} \sigma_{e s}{ }^{4}\right)^{1 / 3}}, \\
& <\delta E>=-0.35 \frac{r_{e} N_{e} L_{e f f}}{\gamma\left(R^{2} \sigma_{e s}{ }^{4}\right)^{1 / 3}},
\end{aligned}
$$

where $L_{e f f}$ is total effective length of bending path, $R$ is the bending radius, $\sigma_{e s}$ is the rms length of the bunch, $N_{e}$ is number of electron per bunch and $r_{e}$ is the classical radius of electron.

Taking typical parameters of the electron beam transport one gets approximately $\sigma_{E}=5 \times 10^{-4}$ and $<\delta E>=-7 \times 10^{-4}$. Both effects are very small in comparison of the energy spread due to long bunch and RF curvature.

Although estimates presented above suggest that CSR effects will be not noticeable, a more accurate 
evaluation of the CSR effect is still needed, including computer simulations of CSR with space charge effects.

More studies need to be done in order to see effects of wake fields and trapped ions. For example in order to minimize the impact of any kind of ports (the beam profile monitors cross, vacuum pump etc) we plan to use cages (see diagnostics for R\&D ERL report).

\section{Future Uses of R\&D ERL and Components}

\section{Coherent Electron Cooling}

After the testing main components of ERL in building 912 with some additional components and a little modifications the $20 \mathrm{MeV}$ R\&D ERL can be moved at RHIC IP2 (or 1IP12) are planning to use to make a coherent electron cooling proof-of-principle experiment to cool $\mathrm{Au}$ ions in RHIC at $\sim 40 \mathrm{GeV} / \mathrm{n}$ (for details of this experiment see [16]). As an example, the layout of coherent electron cooling proofof-principle test installed at RHIC IR2 is shown in Fig.22.

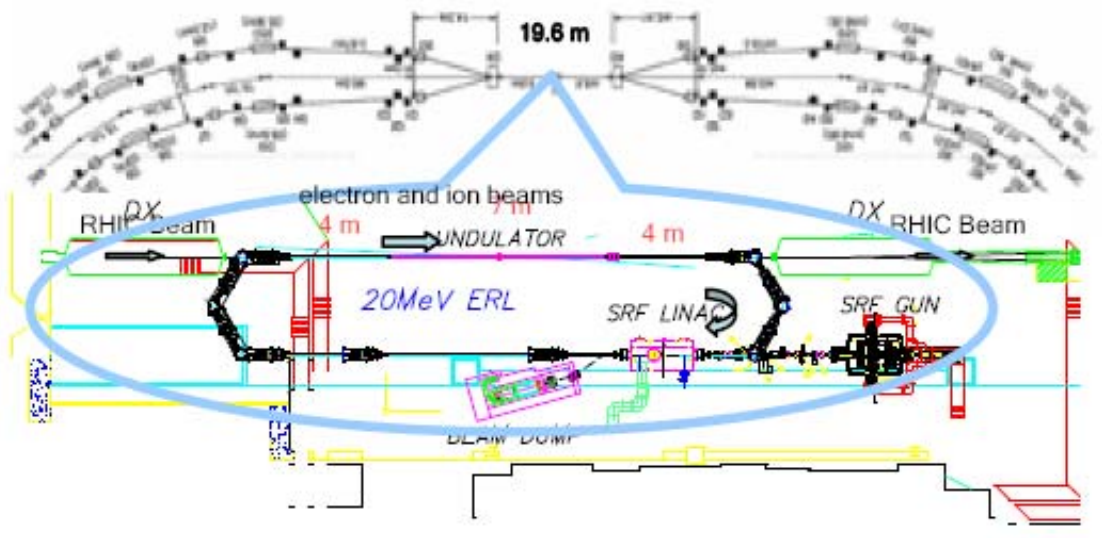

Figure 22: Coherent electron cooling proof-of-principle layout at RHIC IR2.

\section{Conventional or Optics Free FEL}

The availability of high current electron beam with low emittance (Table 3) opens new perspective of using BNL R\&D ERL as an electron beam provider for free electron laser. The simplest upgrade is to install in the dispersion free straight section bypass with one undulator and mirrors from both side. For shorter wavelength we consider the potential extension of this facility into two turn configuration and installation of IR FEL (Fig 23). The shielded vault is designed for ERL with maximum energy of $54 \mathrm{MeV}$ to accommodate these future up-grades. The loop of the ERL is designed to accommodate large energy spread of electron beam in the case of operating a $100 \mathrm{~kW} \mathrm{CW} \mathrm{FEL} \mathrm{[5].}$ 


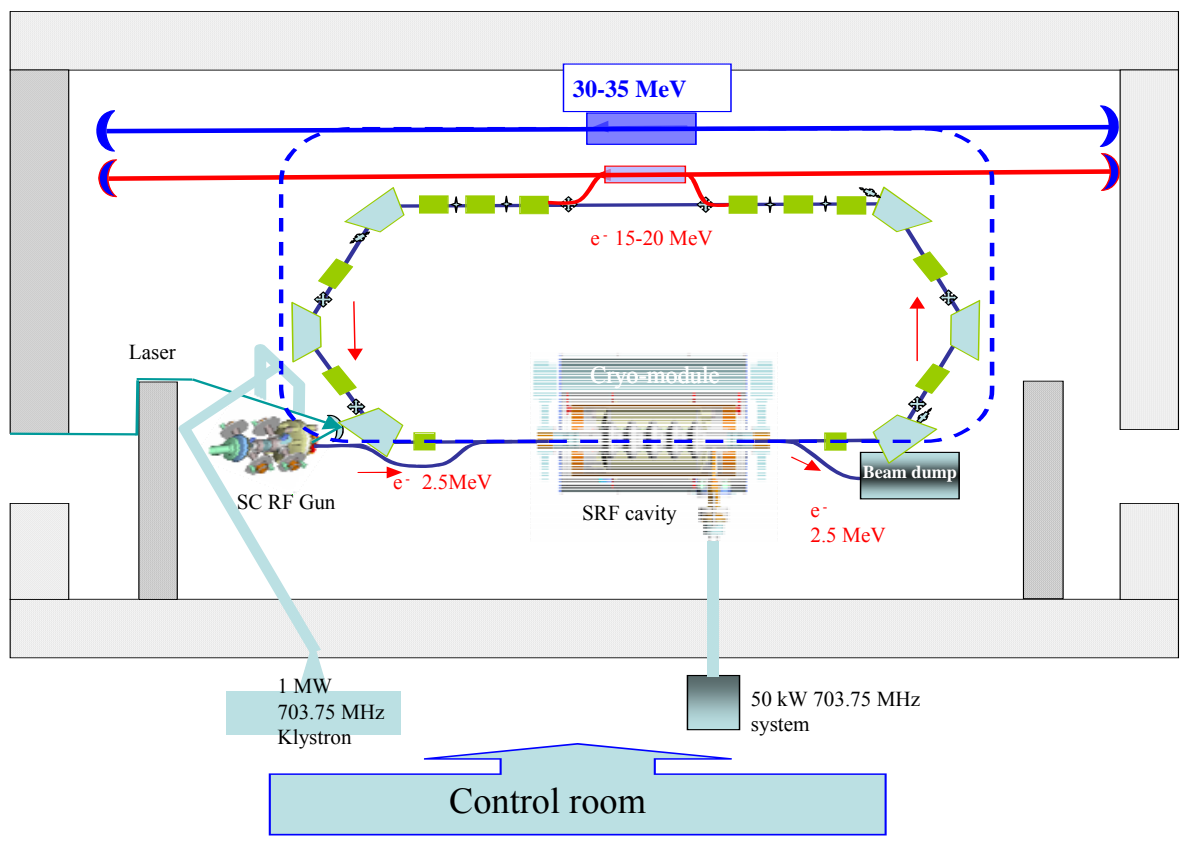

Figure 23: Layout of the R\&D Energy Recovery Linac in the shielded vault with possible FEL setup. Dashed line shows considered second pass up-grade

Other option is to use the high power electron beam in optics-free (ring [17]) FEL. The simple schematic of such type of FEL layout is shown in Fig. 24. There are two undulators in straight section and isochronous 360 degrees bend. Radiation from the second undulator (amplifier) produces energy modulation of the new coming beam inside the first undulator (modulator). The isochronous bend delivers the modulated electron beam in the amplifier. One of the big issues is to preserve micro-bunching structure of the electron beam during the 360 degrees turn. From the first look the micro-bunching can be preserve using arcs with negative bending in the middle.

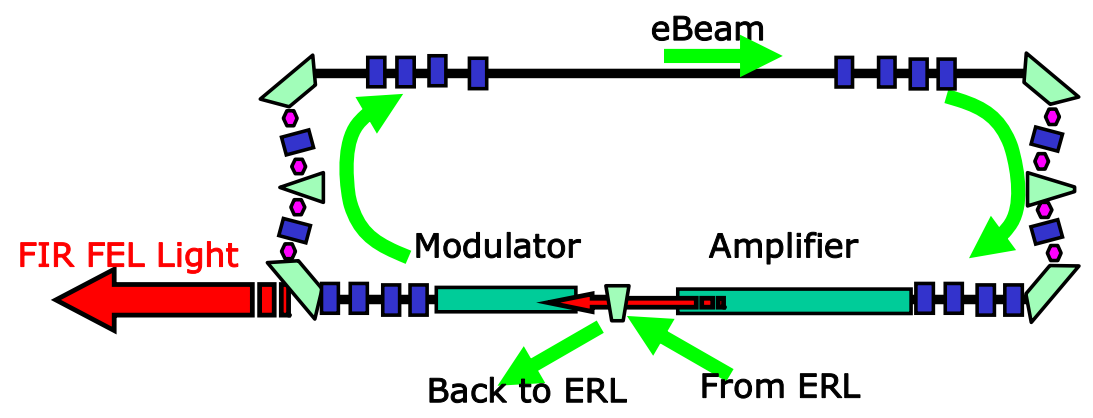

Figure 24: Schematic layout of optics free FEL

For undulators period of $5 \mathrm{~cm}$ and electron bunch of $0.7 \mathrm{nC}$ (Tab. 3) at repetition frequency $9.38 \mathrm{MHz}$ the GENESIS [18] simulation gives: wavelength 29 microns, peak power $2 \mathrm{MW}$ and average power $400 \mathrm{~W}$ (simulation performed by O. Shevchenko BINP, Novosibirsk). For full current mode operation repetition rate $703.75 \mathrm{MHz}$ we obtain $30 \mathrm{~kW}$ far infrared in CW mode. More details about using the BNL R\&D ERL for optical free FEL can be found somewhere else [19].

\section{Conclusions}

We have designed and start commissioning a small (about 20 meters in circumference) R\&D ERL to test the key issues of amp-class CW electron accelerator with high brightness beams, required for future nuclear physics experiments MeRHIC/eRHIC (the foot print of high power ERLs are shown 
on Fig. 25). Extensive R\&D program on many novel components to be used in the ERL is under way. The prototype ERL will demonstrate the main parameters of the electron beam required for electron cooling and for electron ion colliers.

This facility will serve as the test-bet for new range of beam parameters whose application will extend well beyond the goals set forward by Collider Accelerator Department at BNL.

The high average current and nice performance of the electron beam makes BNL R\&D ERL opens the potential of using this machine as an electron source for kilowatts class FELs.

This work is performed under the auspices of the US Department of Energy.

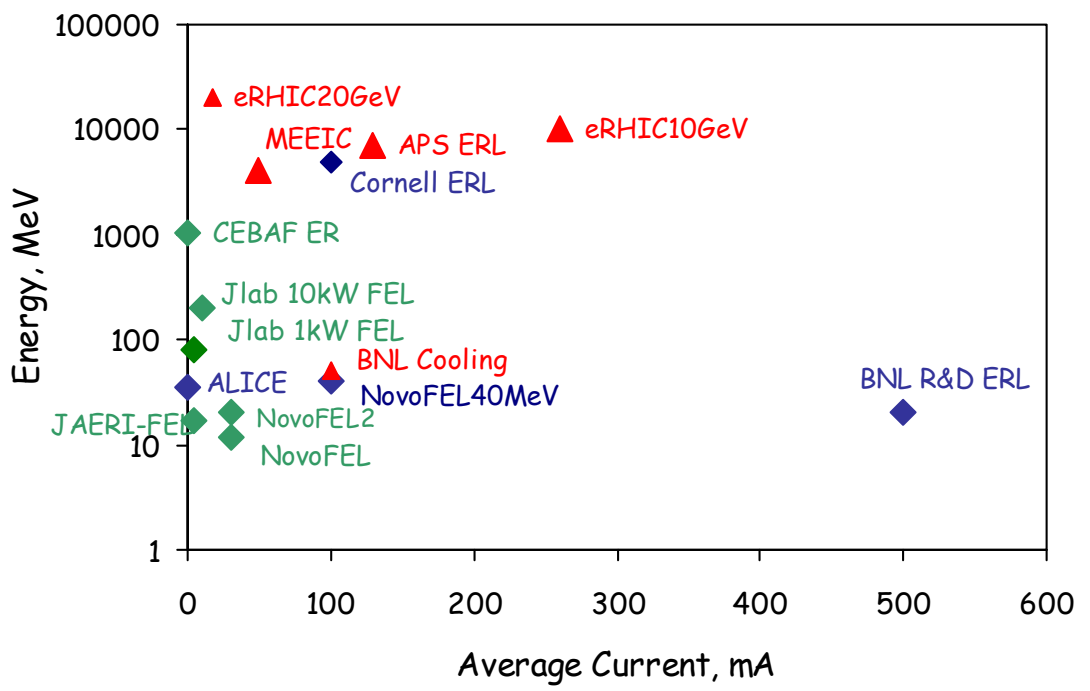

Figure 25: The High Power ERL landscape. Green are already demonstrated/operational, Red under design, Blue under commissioning.

\section{REFERENCES}

[1] I. Ben-Zvi at al., "Extremely High Current, High-Brightness Energy Recovery Linac”, in Proceedings of PAC2005.

"RHIC II FEASIBILITY STUDY", at http://www.bnl.gov/cad/ecooling

[2] T. Hallman, T. Kirk, T. Roser and R.G. Milner, "RHIC II/eRHIC White Paper," Submitted to the NSAC Sub-Committee on Future Facilities, February 15, 2003, http://www.bnl.gov/henp/docs/NSAC RHICII-eRHIC 2-15-03.pdf

[3] Multi-Alkali Photocathode Development at BNL, A. Burrill et al., PAC2005.

[4] V.N. Litvinenko, R. Hajima, D. Kayran, NIM A 557, (2006) pp 165-175.

[5] D. Kayran et al, Optics for High Brightness and High Current ERL Project at BNL, in Proceedings of PAC 2005, pp. 1775-1777.

[6] D. Kayran, V.N. Litvinenko, Merger system optimization in BNL's high current R\&D ERL. in Proceedings of PAC'07, pp. 3711-3713.

[7] A. M. M. Todd et al., "State-of-the-Art Electron Guns and Injector Designs for Energy Recovery Linacs",, in Proceedings of PAC2005, p. 2292.

[8] J. B. Rosenzweig and L. Serafini, Phys. Rev. E 49, 1599 (1994)

[9] L. Young, PARMELA Reference Manual, LA-UR96-1835

[10] Rama Calaga, Ph.D. Thesis Stony Brook University, 2006. 
[11] Wuzheng Meng et al., Unique features in magnet designs for R\&D Energy Recovery Linac at BNL, in Proceedings of PAC07, MOPAS097

[12] E. Pozdeyev, "Compensation of Second Order Dispersion Function in BNL R\&D ERL". Private comunication.

[13] G. A. Krafft and J. J. Bisognano, in Proceedings of PAC 87, Washington, DC, pp. 1356-1358.

[14] E. Pozdeyev, Phys. Rev. ST Accel. Beams 8, 054401 (2005).

[15] H. Hahn et al., "R\&D ERL - HOM ABSORBERS" C-AD AP-notes 369.

[16] V. N. Litvinenko et al., in Proceedings of FEL 2004, p. 570.

[17] N.A. Vinokurov, O.A. Shevchenko, NIM A528 (2004) 491-496.

[18] S. Reiche, NIM A429, (1999) 243.

[19] D. Kayran, et al., FEL potential of the high current ERLs at BNL. in Proceedings of FEL 2007 Novosibirsk, Russia, 2007, pp 232-235 